This increases parental burden and impedes quality of life of both child and parents. A poll amongst parents of children with congenital anatomical anomalies showed a need to better prepare parents for hospital discharge with their chronically ill child. Besides, parents needed a ward providing a more structured environment for child and parents rather than the hectic PICU.

Aim Evaluation of a high dependence transitional care unit (HDTCU) as part of PICU care.

Methods A 6 bedded HDTCU for chronically ill children in need of intensive nursing care was built, managed under full (medical and nursing) responsibility of our PICU. Increasingly during admission, parents take responsibility of caring for the child. We calculated duration of admission and bed occupancy rate. Furthermore a survey was held evaluating satisfaction among parents.

Results Pallieterburght is an extramural HDTCU providing care between PICU and home for children dependent on medical appliances. From January 2010 until April 2012, 33 children were admitted with a median length of stay of 50 days (range 4-345). Bed occupancy was $67 \%$. Main appliances were: tracheal cannula 16 (48\%), mechanical home-ventilation $3(1 \%)$ and home parenteral nutrition 12 (36\%). Parental satisfaction and parental confidence at discharge were high.

Conclusions Although the number of patients is too small to draw definitive conclusions, occupancy rates are high and parents seemed better prepared to accept full responsibility in home-care for their child.

\section{OUTCOME OF CHILDREN ADMITTED TO A REGIONAL PAEDIATRIC INTENSIVE CARE UNIT (PICU) WITH CONVULSIVE STATUS EPILEPTICUS (CSE)}

doi:10.1136/archdischild-2012-302724.0814

S Jayachandran, P Ramesh, R Kumar, Paediatric Intensive Care. Paediatric Intensive Care Unit, University Hospital of North Staffordshire, Stoke-on-Trent, UK

Background and Aims Children presenting with CSE are commonly referred to PICU for ongoing care. Limited data is available on their outcome.

Objectives:

1. To determine mortality and short-term morbidity of children admitted to a regional PICU with CSE

2. To identify differences in outcome for those with preexisting epilepsy compared to non-epileptics.

Methods A retrospective cohort study of 57 children (1 month to 16 years) admitted to a regional PICU between January 2008 and December 2011 with CSE was carried out. Study population were divided into Group 1 ( $\mathrm{n}=23$, presence of pre-existing epilepsy) and Group 2 ( $n=34$, absence of pre-existing epilepsy).

Results The results of the group 1 and 2 respectively were:

Median age (months): 33 and 21.5

Male: female ratio (\%): $57: 43$ and $76: 24$

Refractory seizures ( $>60 \mathrm{~min}$ ) at presentation: $83 \%$ and $53 \%$ Deviation from APLS protocol for seizure control: $43 \%$ and $32 \%$

Extra doses of benzodiazepine: 50\% and 91\%

Neurological co-morbidity:91\% and 24\%

Median duration of PICU ventilation: $18.1 \mathrm{hrs}$ and $10.5 \mathrm{hrs}$

Median duration of PICU stay:24.1 and $24.5 \mathrm{hrs}$

Seizure control at PICU admission: $61 \%$ and $94 \%$

Midazolam infusion in PICU: $96 \%$ and $97 \%$

Neurological investigations (LP, CT/MRI Head, EEG) performed in $22 \%$ and $94 \%$

Pre-discharge neurological morbidity: 2 (aggressive behaviour, decerebrate posturing) and 1 (increased tone)

No mortality identified
Abstract 814 Table 1

\begin{tabular}{lll}
\hline Percentage & Group 1 & Group 2 \\
\hline Median age (months) & 33 & 21.5 \\
Male:Female & $57: 43$ & $76: 24$ \\
Refractory seizures $>$ 60min & 83 & 53 \\
APLS protocol for seizure control deviation & 43 & 32 \\
Reason for deviation: extra doses of & 50 & 91 \\
benzodiazepine & & \\
Neurological co-morbidity & 91 & 24 \\
PICU ventilation (median hours) & 18.1 & 10.5 \\
PICU stay (median hours) & 24.1 & 24.5 \\
\hline
\end{tabular}

Abstract 814 Table 2 PICU Events

\begin{tabular}{lll}
\hline & Group 1 & Group 2 \\
\hline Seizure control at PICU admission & $61 \%$ & $94 \%$ \\
Midazolam infusion in PICU & $96 \%$ & $97 \%$ \\
Neurological investigations (LP, CT/MRI Head, & $22 \%$ & $94 \%$ \\
EEG) & \multicolumn{2}{l}{} \\
Neurological morbidity & 2 (aggressive behaviour, 1 (increased tone) \\
Mortality & decerebrate posturing) \\
\hline
\end{tabular}

Conclusion Epileptics presented frequently with refractory seizures and were ventilated longer though this didn't affect total PICU stay; non-epileptics were investigated more frequently. Morbidity $(5 \%)$ was still seen though there was no mortality in our study.

\section{PATIENTS WITH PRIMARY IMMUNODEFICIENCY DISORDERS IN PEDIATRIC INTENSIVE CARE UNIT: OUTCOMES AND MORTALITY-ASSOCIATED RISK FACTORS}

doi:10.1136/archdischild-2012-302724.0815

${ }^{1} \mathrm{C}$ Ödek, ${ }^{1} \mathrm{~T}$ Kendirli, ${ }^{1} \mathrm{G}$ Vatansever, ${ }^{2} \mathrm{~F}$ Doğu, ${ }^{2} \mathrm{~A}$ I kincioğulları. ${ }^{1}$ Pediatric Critical Care; 2Pediatric Immunology and Allergy, Ankara University, Faculty of Medicine, Ankara, Turkey

Introduction Primary immunodeficiency disorders (PID) are characterized by poor or absent function in one or more components of the immune system. Early diagnosis and treatment is crucial for preventing morbidity and mortality. Despite adequate treatment, most of these patients require intensive care because of organ dysfunctions related to infections and HSCT complications. We reviewed our PID patients admitted to pediatric intensive care unit (PICU) over a 10-year period.

Patients and methods: PID patients, who were admitted to PICU between 1 January 2002 and 1 January 2012, were included. Data were collected from patient medical records.

Results A total of 51 patients $(27$ males) were admitted to PICU. There were a total of 71 admission episodes. The median age was 12 months. Age and sex were not significantly associated with mortality. The most common diagnosis was SCID. A total of 20 patients underwent HSCT. In all, 52 (73.2\%) of all admission episodes were for respiratory problems, 10 (14\%) for proven infections, 8 (11.4\%) for neurological problems and 1 (1.4\%) for surgical problems. Of the 71 episodes, $51(71.8 \%)$ required mechanical ventilation, $11(15.4 \%)$ required renal replacement therapy, $32(45 \%)$ required inotropes. In all, 40/71 (56.3\%) of the episodes resulted in survival. Requirement for ventilation, inotropes and renal replacement therapy were related with poor outcome. Multi-organ failure, PELOD score, duration of PICU admission were associated with mortality. 
Conclusion Patients with PID had a high rate of PICU admission. Multi-organ failure was associated with mortality. Despite adequte support mortality rates were around $50 \%$.

\section{PULMONARY HAEMORRHAGE IN VERY LOW BIRTH WEIGHT INFANTS: 10 YEARS EXPERIENCE IN TERTIARY NICU}

doi:10.1136/archdischild-2012-302724.0816

${ }^{1} \mathrm{~A}$ Nosherwan, ${ }^{1} \mathrm{~K}$ Bermijo, 'D O'Leary, 1,2,3E Molloy. 'Neonatology, National Maternity Hospital; ${ }^{2}$ Paediatrics, University College Dublin; ${ }^{3}$ Paediatrics, Royal College of Surgeons in Ireland, Dublin, Ireland

Aims To study the clinical course, associated neonatal morbidity and mortality following pulmonary haemorrhage $(\mathrm{PH})$ in VLBW infants.

Methods Infants $<1500$ gms that developed pulmonary haemorrhage were identified from the hospital database. Outcome was measured as death or survival following acute $\mathrm{PH}$.

Results Total of 1206 VLBW infants were admitted in our NICU between 2002 to 2011. Incidence of $\mathrm{PH}$ was 3\%(n=40) in this group. Mean gestational age (SD) was $26 \pm 1.8$ weeks with mean Birth weight of $0.8 \pm 0.2 \mathrm{~kg}$. Cause of preterm delivery was fetal distress $n=7$, maternal PET $n=8$, maternal sepsis $n=10$ and preterm labour $n=10$. Eleven of those mothers received antenatal steroids. Antenatal steroids were associated with improved survival $(p<0.001)$. Eight babies had evidence of intrauterine growth retardation and six babies were septic at birth. Five patients required cardiopulmonary resuscitation at birth. The mean age at $\mathrm{PH}$ was $47.7 \pm 28$ hours. Sixteen patients received prophylactic surfactant. Two patients had mild, nine had moderate and twenty-nine had severe $\mathrm{PH}$. Ten cases were treated with surfactant with acute PH. Half of the infants had a PDA. Eighteen patients were coagulopathic at the time of $\mathrm{PH}$. Twenty-one babies died (53\%) and none had received antenatal steroids. In the surviving infants, ten developed CLD and six had Mental Developmental Index $<70$.

Conclusion Our institutional incidence of pulmonary haemorrhage and outcome is similar to other reports in the literature. Comprehensive evidence base management strategies are required to standardise care of the VLBW infants who develop $\mathrm{PH}$.

\section{EARLY NEONATAL OUTCOME IN NEWBORNS EXPOSED TO CHEMOTHERAPY DURING FETAL PERIOD}

doi:10.1136/archdischild-2012-302724.0817

'M Fumagalli, 'L Bassi, ${ }^{2} \mathrm{G}$ Scarfone, ${ }^{2} \mathrm{~F}$ Peccatori, 'S Pisoni, 'S Passera, 'M Groppo, 'A De Carli, 'I Sirgiovanni, 'F Dessimone, 'F Mosca. 'NICU, Fondazione IRCCS Ca' Granda, Ospedale Maggiore Policlinico University of Milan; ${ }^{2}$ Second Obstetric and Gynecologic Clinic, University of Milan and Fondazione IRCCS Ca' Granda Ospedale Maggiore Policlinico, Milan, Italy
Introduction Cancer treatment in pregnant women is still a matter of debate as life-saving therapies for the mother and adverse effects for the fetus. Recent data support that chemotherapy from 14 wks GA onwards as safe in terms of early neonatal and long-term outcomes in children, while impaired neurodevelopmental outcome appear to be related to prematurity.

Aim To describe the early neonatal outcome in newborns exposed to chemotherapy during pregnancy.

Methods Clinical data of inborn babies, born between 2005 and 2011, from mother treated with chemotherapy, were retrospectively collected from clinical notes.

Results In our population of neonates exposed to chemotherapy during pregnancy 18\% (4/22) were born between 25 and 33 weeks of GA and 73\% (16/22) between 34 and 36 wks, 9\% at term, $(2 / 22)$. The $70 \%$ was admitted to post-natal ward and the $95 \%$ was AGA, while only $5 \%$ SGA. Neonatal complications observed were: respiratory distress syndrome 32\% (7/22; 3/7 CPAP, 3/7 meccanical ventilation), hypoglycemia $23 \%(5 / 22)$ and jaundice $23 \%(5 / 22)$. Two very preterm babies $(<27 \mathrm{wks})$ developed intraventricular haemorrhage. Not cardiac complications and not significant hematological abnormalities (neutropenia, severe acute anemia and low platelet count) were observed in the neonatal period.

Conclusions According to our data chemotherapy during pregnancy doesn't seem to influence early neonatal outcome. Otherwise more studies are needed to confirm the safety of cancer treatment during pregnancy in terms of long-term cognitive and neurobehavioural outcomes in order to define treatment strategies aimed to reduce iatrogenic preterm birth.

\section{LENGTH OF HOSPITAL STAY (LOS) OF NEONATES UNDERGOING SURGERY AT A TERTIARY NEONATAL UNIT}

doi:10.1136/archdischild-2012-302724.0818

${ }^{1}$ SK Shetty, 'N Kennea, ${ }^{2}$ S Giuliani, 'P Desai, '1 Richards. 'Neonatal Unit; ${ }^{2}$ Paediatric Surgery Unit, St George's Hospital NHS Trust, London, UK

Background and Aims Detailed length of stay (LOS) data for infants following surgical procedures are important for prediction of neonatal resources and helpful when counselling parents following a new diagnosis. There are few recent data available on LOS for different surgical conditions in the literature, and none describing a range of procedures from one centre.

Objective To describe LOS for common surgical procedures in a single centre tertiary referral neonatal unit.

Methods Data were collected for a 5-year period. If babies were transferred to another unit following surgery, these units were contacted to determine total LOS. We included babies that had surgery during their first admission to our unit and who survived to discharge.

Results 